\title{
VALORACIÓN DEL ORIGEN AFRICANIZADO EN LA INTEGRACIÓN DE UNA POBLACIÓN EXPERIMENTAL DE Apis mellifera L.
}

\author{
ASSESSMENT OF THE AFRICANIZED ORIGIN IN THE INTEGRATION \\ OF AN EXPERIMENTAL POPULATION OF Apis mellifera L.
}

\section{Rebeca A. Urbina-Romero ${ }^{*}$, Fernando Utrera-Quintana ${ }^{2}$, Fernando Castillo-González ${ }^{1}$, Manuel Livera-Muñoz ${ }^{1}$, Ignacio Benítez-Riquelme ${ }^{1}$, Abel E. Villa-Mancera ${ }^{2}$, Jorge E. Hernández-Hernández ${ }^{2}$ e Hilda V. Silva-Rojas ${ }^{3}$}

\begin{abstract}
'Colegio de Postgraduados (COLPOS), Postgrado en Recursos Genéticos y Productividad-Genética, Montecillo, Texcoco, Estado de México, México. ${ }^{2}$ Benemérita Universidad Autónoma de Puebla, Facultad de Medicina Veterinaria y Zootecnia, Tecamachalco, Puebla, México. ${ }^{3} \mathrm{COLPOS}$, Postgrado en Recursos Genéticos y Productividad-Producción de Semillas, Montecillo, Texcoco, Estado de México, México.
\end{abstract}

*Autor para correspondencia (urbina.rebeca@colpos.mx)

\section{RESUMEN}

La abeja africana (Apis mellifera scutellata) se introdujo del sur de África a Brasil en 1956 para inducir recombinaciones genéticas y generar segregantes adaptados a zonas tropicales; sin embargo, 26 colonias escaparon y con ello se inició un proceso de africanización en el continente americano. Se integró un apiario experimental mediante la recombinación de germoplasma europeo y africanizado con propósitos de mejoramiento genético, el cual se ha mantenido sin aplicación de acaricidas contra varroasis por más de 20 años. La población se generó mediante cruzamientos controlados de abejas europeas (Apis mellifera ligustica) que habían estado bajo mejoramiento genético por alrededor de 15 años (fuente de zánganos) con abejas africanizadas ( $A$. $m$. scutellata con probable recombinación con diversas subespecies de abejas europeas) que habían estado sujetas a un proceso de selección para su semidomesticación y mejoramiento genético bajo criterios apícolas (fuente de abejas reinas). Con el objeto de tener información sobre el origen materno de la población, se realizó secuenciación de las regiones intergénicas COICOll y ND5 del $A_{D N}$ en una muestra aleatoria de 19 colmenas. Se confirmó el origen materno africano de $A$. m. scutellata $(74 \%)$ y se reveló la presencia de colmenas de origen híbrido de $A$. m. scutellata $\times A$. m. capensis $(26 \%)$, no reportado en México hasta antes de esta investigación. Los resultados parece indicar que las abejas africanizadas han conservado su ADN mitocondrial a través del proceso de dispersión.

Palabras clave: Apis mellifera, ADN $_{\text {mtt }^{\prime}}$ abeja africanizada, euroafricana, COI-COII, ND5.

\section{SUMMARY}

The African bee (Apis mellifera scutellata) was introduced from South Africa to Brazil in 1956 to induce genetic recombinations and generate segregants adapted to tropical areas; however, 26 colonies escaped and a process of Africanization started in the American continent. An experimental apiary was integrated by recombination of European and Africanized germplasm for breeding purposes, which has been maintained without application of acaricide against varroasis for more than 20 years. The population was generated by controlled mating of European bees (Apis mellifera ligustica) that had been under genetic improvement for about 15 years (source of drones) with Africanized bees (A. m. scutellata with probable genetic recombination with other European subspecies) that had been under to a selection process for its semi-domestication and genetic improvement under apicultural criteria (source of queen bees). In order to obtain information on the maternal origin of the population, the intergenic regions COI-COII and ND5 of the ${ }_{\mathrm{mt}} \mathrm{DNA}$ were sequenced in a random sample of 19 hives. The maternal African origin of $A$. m. scutellata $(74 \%)$ was confirmed and the presence of hives of hybrid origin of $A$. $m$. scutellata $\times A$. m. capensis (26\%), not reported in Mexico before this investigation, was revealed. This seems to indicate that Africanized bees have conserved their mitochondrial DNA through the dispersion process.

Index words: Apis mellifera, ${ }_{\mathrm{mt}} \mathrm{DNA}$, Africanized honeybees, EuroAfrican, COI-COII, ND5

\section{INTRODUCCIÓN}

La subespecie Apis mellifera scutellata se introdujo del sur de África a Brasil en 1956 para realizar recombinaciones genéticas y generar segregantes adaptados a zonas tropicales (Kerr, 1967); sin embargo, en 26 colonias escaparon en 1957 y con ello se inició un proceso de africanización en el continente americano (Kerr, 1967; Padilla et al., 1992). La abeja africanizada se reconoce como el resultado del apareamiento, en primera instancia, de reinas de origen europeo de apiarios locales con zánganos de origen africanizado escapados del Brasil, y en segunda instancia la invasión de colmenas por reinas de origen africanizado (Clarke et al., 2002); la dispersión hacia el norte se da con esta dinámica y a finales de 1986 se detectaron los primeros enjambres en México (Winston, 1992), lo que ha provocado pérdidas económicas en la apicultura por su comportamiento altamente defensivo, migratorio y fuerte tendencia a enjambrar (Guzmán-Novoa y Page, 1994a; Seeley, 2016; Stort, 1974).

En 1984 se creó el Programa Nacional para el Control de la Abeja Africana para prevenir y controlar su diseminación en México. La estrategia que se planteó para hacer frente a la invasión de la abeja africanizada consistió en establecer trampas y su consecuente eliminación y así preservar los apiarios locales con germoplasma europeo (Cajero, 1990,

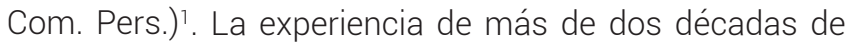

${ }^{1}$ Cajero A. S. (1990) Avances en las acciones para el control de la abeja africana en México, antecedentes. In: Memorias 3er. Seminario Americano de Apicultura. Acapulco, Gro. México. p:1. 
dispersión mostró que las trampas fueron inefectivas y la abeja continuó su dispersión a través de territorio mexicano y en 1990 se localizó en Texas, EE. UU. en 1990 (Sugden y Williams, 1991), por lo que el programa de control de la abeja africanizada terminó siendo sólo de monitoreo de su avance en el territorio mexicano (Winston, 1992).

Una estrategia alternativa la planteó el Dr. Tarcicio Cervantes-Santana, quien en 1988 inició un programa de mejoramiento genético, el cual consideró el aprovechamiento de la variabilidad genética que representaba la incorporación de genes de abejas africanizadas. El apiario experimental del Colegio de Postgraduados, Campus Montecillo, Estado de México se integró en 1978 inicialmente con abejas reinas europeas colectadas de diferentes partes del país, para aplicar selección con criterios de sanidad y buen comportamiento apícola. En 1993 se introdujeron al apiario abejas africanizadas que habían estado sometidas a un proceso de semidomesticación mediante selección en Cárdenas, Tabasco por cuatro generaciones, para mansedumbre, producción de miel y bajo comportamiento de enjambrazón y migración (Cervantes y Cruz, 1997, Com. Pers.)2. Se criaron reinas de las mejores colmenas africanizadas seleccionadas, las cuales se aparearon a través de cruzamientos controlados con zánganos europeos mejorados provenientes de las colmenas iniciales.

Derivado de este proceso y más de 20 años de selección continua, en el Colegio de Postgraduados se cuenta con una población de abejas denominadas euroafricanas (Pérez-Sato y Cervantes-Santana, 2001), de diferente constitución a las africanizadas mencionadas por otros autores (Schneider et al., 2004), Ilamadas euroafricanas por la recombinación genética de abejas reinas africanizadas seleccionadas con zánganos europeos (Utrera, 1998, Com. Pers.) $)^{3}$.

Aunque se conoce la manera en que se construyó el acervo genético de la población, no se tiene información a nivel de ADN que respalde su naturaleza genética, por lo que es importante realizar estudios que permitan tener información confiable sobre el origen materno de este material genético. Es conveniente señalar que este apiario experimental se ha mantenido sin aplicar ningún tipo de tratamiento contra la varroasis por más de 20 años, capacidad que puede ser atribuible a la contribución del germoplasma africanizado con la mejora genética señalada.

${ }^{2}$ Cervantes S. T. y A. M. Cruz (1997) Avances en la domesticación y el mejoramiento de abejas africanas. In: Memorias XI Seminario Americano de Apicultura. Acapulco, Guerrero, México. Septiembre de 1997. pp:34-36.

${ }^{3}$ Utrera Q. F. (1998) Análisis de la transmisión a la descendencia de la tolerancia a Varroa jacobsoni O., de una población de abejas. Tesis de Maestría en Ciencias. Colegio de Postgraduados. Montecillo, Edo. de México. 60 p.
Anteriormente, era difícil distinguir a la abeja africanizada de las subespecies europeas debido a la homogeneidad en la morfología de la especie (Gary et al., 1985); sin embargo, hoy en día existen métodos eficientes para detectar la africanización, como la amplificación de fragmentos de $A D N_{m t^{\prime}}$ debido a que éste se hereda vía materna y revela la identidad genética de la reina a través de las obreras sin tomar en cuenta la herencia paterna (Hall y Muralidharan, 1989). Con información de morfometría, de polimorfismo isoenzimático y de $\mathrm{ADN}_{\mathrm{mt}}$ actualmente se reconocen 29 subespecies de $A$. mellifera, que se agrupan en cinco linajes: linaje A, incluye a las subespecies africanas; linaje $M$, formado por subespecies de Europa occidental; linaje C, formado por las subespecies de Europa oriental; linaje O, que abarca a las subespecies del cercano oriente, y linaje $Y$, que incluye a la subespecie de Etiopía (Özdil y İlhan, 2012; Rahimi, 2015; Ruttner, 1988; Valido et al., 2014).

Por lo anterior, el objetivo del presente estudio fue determinar el origen materno en el apiario mediante el análisis de las regiones intergénicas COI-COIl y ND5 del $\mathrm{ADN}_{\mathrm{mt}}$ con la expectativa de que corresponde al linaje africano que se empleó en el establecimiento de la población experimental.

\section{MATERIALES Y MÉTODOS}

\section{Sitio experimental}

La investigación se realizó durante los meses de febrero a julio de 2015 en el apiario experimental del Colegio de Postgraduados, Campus Montecillo, ubicado en Texcoco, Estado de México (19² 27' 50.01" latitud N, $98^{\circ}$ 54' 17.53" longitud $0,2,250 \mathrm{msnm}$ ). El clima predominante es templado semi-seco, con temperatura media anual de $15.9^{\circ} \mathrm{C}$, y heladas poco frecuentes en otoño-invierno.

\section{Material genético}

La población provino de cruzas efectuadas en Montecillo, Edo. de México en 1993, de abejas europeas mejoradas con abejas africanizadas que habían sido sujetas a un proceso de selección para su semidomesticación (Guzmán-Novoa, 1996; Pérez-Sato y Cervantes-Santana, 2001). Las abejas africanizadas procedieron de una población en proceso de mejoramiento genético en Cárdenas, Tabasco, México. La población de abejas africanizadas se formó con enjambres recolectados en Tabasco, Campeche y Veracruz en 1988. Esta población de enjambres fue seleccionada durante cuatro generaciones, una por año, para los caracteres de mansedumbre, sedentarismo y productividad apícola mediante la cría artificial de reinas y fecundación natural. 
La población de abejas europeas, también mejoradas, se integró de la siguiente forma: 1) en 1978 se introdujeron reinas europeas fecundadas provenientes de 10 localidades de México al apiario en Chapingo, Texcoco, Edo. de México, para criar reinas hijas; 2) la progenie resultantes se fecundó en apareamiento natural para obtener la primera generación recombinante; 3) después de dos generaciones de recombinación, la población se seleccionó durante 13 generaciones (una por año) hacia características asociadas con la productividad de miel, tales como sanidad y uniformidad de postura, mediante cría artificial de reinas y con apareamiento natural; posteriormente, el apiario se trasladó de Chapingo a Montecillo, Texcoco, Edo. de México.

En 1993 se tomaron 20 reinas africanizadas seleccionadas para mansedumbre, baja enjambrazón y productividad apícola de Cárdenas, Tabasco, mismas que se introdujeron al apiario de Montecillo, integrado con colonias de abejas europeas mejoradas. De las reinas africanizadas introducidas se seleccionaron seis, cuyas colonias presentaron los mejores atributos apícolas, tales como mansedumbre, calidad de postura, desarrollo en su población y productividad de miel. De estas reinas se obtuvieron reinas hijas por cría artificial, las cuales se fecundaron en forma natural con zánganos europeos que predominaban en el tiempo referido. Las cruzas entre reinas vírgenes africanizadas con zánganos africanizados se evitaron colocando trampas en la piquera de cada colmena y destruyendo pupas de zánganos. La población resultante se mantuvo bajo selección durante tres ciclos para docilidad, calidad de postura y producción de miel. Este proceso de selección se mantuvo de manera permanente por las generaciones correspondientes a uno por año hasta el inicio de este estudio.

\section{Análisis genético}

Se eligieron 19 colmenas al azar para su valoración molecular de las más de 100 colmenas tipo jumbo que integran el apiario. Se colectaron al menos 30 obreras de cada una de las 19 colonias, las cuales se depositaron en frascos de plástico para ser conservadas a $-20^{\circ} \mathrm{C}$ hasta su uso. El ADN total se extrajo del cuerpo completo de cada abeja obrera (cinco individuos por colmena) con el método CTAB (Doyle y Doyle, 1987). El análisis del $\mathrm{ADN}_{\mathrm{mt}}$ se llevó a cabo mediante la amplificación por reacción en cadena de la polimerasa (PCR) de las regiones intergénicas COICOll y ND5, según el protocolo descrito por Garnery et al. (1992). Los iniciadores utilizados para COI-COII fueron: E2 forward 5'-GGCAGAATAAGTGCATTG-3' y H2 reverse 5'-CAATATCATTGATGACC-3', que amplifican un fragmento de 530 a 1230 pb (Magnus et al., 2014); para el gen ND5 los iniciadores fueron: ND5 forward
5'-CGAAATGAATAGGATACAG-3' y ND5 reverse 5'-TTAGGATTTGGTAGAGTTGG-3', que amplifican un fragmento de aproximadamente 822 pb (Bouga et al., 2005; Özdil y illhan, 2012).

La mezcla de reacción de PCR se preparó en un volumen final de $15 \mu \mathrm{L}$, integrado por $7.86 \mu \mathrm{L}$ de agua HPLC, 3 $\mu \mathrm{L}$ de buffer, $0.6 \mu \mathrm{L}$ de dNTPs, 0.18 de cada iniciador, 3 $\mu \mathrm{L}$ de ADN genómico y $0.18 \mu \mathrm{L}$ de Taq ADN polimerasa (Promega, USA). Las condiciones de amplificación de PCR para COI-COII fueron una desnaturalización inicial a $94{ }^{\circ} \mathrm{C}$ por 4 min, 40 ciclos de $94{ }^{\circ} \mathrm{C}$ por $1 \mathrm{~min}, 48{ }^{\circ} \mathrm{C}$ por 1 min y $72{ }^{\circ} \mathrm{C}$ por 1 min, y una extensión final de $72{ }^{\circ} \mathrm{C}$ por 6 min; y para ND5 una desnaturalización inicial a $94^{\circ} \mathrm{C}$ por 5 min, 35 ciclos de $94{ }^{\circ} \mathrm{C}$ por $45 \mathrm{~s}, 46^{\circ} \mathrm{C}$ por 1 min y 72 ${ }^{\circ} \mathrm{C}$ por 1 min, y una extensión final a $72{ }^{\circ} \mathrm{C}$ por 5 min. La amplificación de los genes se verificó en geles de agarosa $1.5 \%$ con amortiguador 1X TAE. El producto final de PCR se purificó con ExoSAP-IT Affymetrix®. La secuenciación se realizó en ambas direcciones con un secuenciador Genetic Analyzer modelo $3230 \AA$ (Applied Biosystem ${ }^{\circledR}$, Foster City, California, EUA).

\section{Análisis de la información}

El ensamble de secuencias se obtuvo mediante el programa de cómputo BioEdit versión 7.0.5 (Hall, 1999) y éstas fueron alineadas con el procedimiento Profile Mode Muscle (Thompson et al., 1994) incluido en el programa Mega6 (Tamura et al., 2013). Las secuencias consenso fueron comparadas con las depositadas en el GenBank del National Center for Biotechnology Information (NCBI) (https://www.ncbi.nlm.nih.gov/genbank/), mediante la opción BLASTN 2.2.19 (Zhang et al., 2000). Se obtuvieron 95 secuencias en total, de las cuales 50 se utilizaron para el análisis Bayesiano.

Las regiones COI-COll y ND5 se concatenaron para realizar un análisis filogenético Bayesiano mediante el software MrBayes v.3.2.0 (Ronquist y Huelsenbeck, 2003); el número de generaciones de cadenas de Markov de Montecarlo (MCMC) fue de $3 \times 10^{6}$, con un muestreo cada 1000 generaciones y una desviación estándar final de 0.009608. El árbol filogenético se construyó utilizando la secuencia del abejorro (Bombus terrestris) como grupo externo, tomada de la base de datos de secuencias depositadas en el Genbank (número de accesión DQ870926.1) y como testigos se utilizaron secuencias de genomas mitocondriales de miembros de los linajes A (africano) y M (europeo): KJ601784 A. m. scutellata (A), KX870183 A. m. capensis (A), KX943034 A. m. scutellata $x$ capensis(A), KT279826 A. m. scutellata (A), KY926883 A. m. intermisa (A), KX463941 A. m. iberiensis (M) y KY926884 A. m. mellifera (M). 


\section{RESULTADOS Y DISCUSIÓN}

\section{Presencia de polimorfismos y agrupamiento}

La longitud de las secuencias concatenadas de las regiones intergénicas ND5 y COI-COII varió de 993 a 1235 $\mathrm{pb}$, derivado de inserciones y deleciones en los fragmentos analizados (Cuadro 1). El análisis concatenado produjo un dendrograma que permitió separar las colmenas en estudio, con base en las secuencias analizadas y tomando como grupo externo a la secuencia de $B$. terrestris, lo que dio como resultado una topología de seis subclados (Figura $1)$, de los cuales, en cinco (I, II, III, IV, VI) se agruparon las 19 colmenas analizadas y uno $(V)$ quedó conformado por tres testigos: A. m. iberiensis (M), A. m. intermisa (A) y A. m. mellifera (M).

El subclado I agrupó secuencias de ocho ejemplares, pertenecientes a cinco colmenas (C51, C65, C23, C45 y C36), así como a las secuencias de tres testigos: el híbrido A. m. scutellata $\times$ A. m. capensis, A. m. capensis y A. m. scutellata; estos testigos fueron caracterizados como linaje $A$, de origen africano. Dicho grupo se distinguió por presentar una deleción de 191 pb con respecto al testigo africano A. m. scutellata (KJ601784, africanizada colectada en Querétaro, México), manteniendo el $84.2 \%$ de los sitios conservados y dos sitios variables.

El subclado II agrupó a dos ejemplares de una colmena, C63, con una inserción de 4 pb con respecto al testigo africanizado anterior. El subclado III, agrupó a 26 ejemplares de nueve colmenas (C01, C31, C60, C77, C80, C111, C117, C128, C177). Este grupo tuvo una deleción de 2 pb y 99.8 $\%$ de los sitios conservados con relación al testigo A. m. scutellata (KJ601784, africanizada colectada en Querétaro, México). El subclado IV, agrupó a dos ejemplares, uno de cada una de dos colmenas (C80 y C77) y al testigo A. m. scutellata (KT279826, africanizada colectada en California, EE. UU.). Este último presentó $99.83 \%$ de sitios conservados en su secuencia y una inserción de 2 pb con relación al testigo A. m. scutellata (KJ601784, africanizada colectada en Querétaro, México), de acuerdo con la base de datos del GenBank.

El subclado $V$ agrupó a tres testigos: A. $m$. iberiensis (KX463941, europeo, linaje $M$, proveniente de Portugal), A. $m$. intermissa (KY926883, africano, linaje A, proveniente de Marruecos) y A. m. mellifera (KY926884, europeo, linaje $M$, proveniente de Noruega), que difirieron por presentar una inserción de 44 pb, una deleción de 159 pb y una deleción 163 pb, respectivamente, con respecto al testigo africanizado (KJ601784, africanizada colectada en Querétaro, México); además de las inserciones/deleciones, se detectó el 0.32, 2.11 y $2.6 \%$ de sitios variables con respecto al testigo africanizado de referencia (Cuadro 2). El subclado $\mathrm{VI}$ agrupó a 12 ejemplares de ocho colmenas (C63, C187, C57, C74, C187, C56, C57, C89), las cuales presentaron una deleción de 240 pb y dos sitios variables $(0.16 \%)$ con relación al testigo A. m. scutellata de Querétaro, México.

El subclado VI agrupó a los dos testigos del linaje europeo $\mathrm{M}$, así como a un testigo africano del linaje $\mathrm{A}$, pero que provino de Marruecos, en el norte de África, además de B. terrestris, como testigo de grupo externo. Este subclado se separó de manera relevante del resto de unidades de estudio, con magnitud de 0.3 unidades de tasa de sustitución por sitio, determinadas por las deleciones/inserciones, así como por la mayor diferencia en sitios variables, lo cual permite aseverar que el material de estudio presenta $\mathrm{ADN}_{\mathrm{mt}}$ de origen africano.

La mayor parte del material de estudio (14 de 19 colmenas) formó subclados con testigos africanizados colectados en México y en California; también, en la comparación con la base de datos del GenBank se corroboró la identidad con A. m. scutellata. Las cinco colmenas restantes se identificaron como híbridos de $A$. $m$. scutellata $\times$ A. m. capensis.

Cuadro 1. Diferencia en polimorfismo en secuencias concatenadas de los espacios intergénicos COI-COII y ND5 de 19 colmenas del apiario experimental del Colegio de Postgraduados, Campus Montecillo, Estado de México.

\begin{tabular}{lccccc} 
Subclado & $\begin{array}{c}\text { No. de } \\
\text { secuencias }\end{array}$ & $\begin{array}{c}\text { Longitud de } \\
\text { secuencias }(\mathrm{pb})\end{array}$ & $\begin{array}{c}\text { Proporción de sitios } \\
\text { conservados }\end{array}$ & $\begin{array}{c}\text { Proporción de } \\
\text { sitios variables }\end{array}$ & $\begin{array}{c}\text { Inserciones/deleciones con respecto a } \\
\text { A. m. scutellata KJ601784 }\end{array}$ \\
\hline I & 11 & 1041 & $1039 / 1233(84.2 \%)$ & $2 / 1233(0.16 \%)$ & Deleción $191 \mathrm{pb}$ \\
II & 2 & 1234 & $1232 / 1235(99.7 \%)$ & $3 / 1235(0.24 \%)$ & Inserción $4 \mathrm{pb}$ \\
III & 26 & 1234 & $1232 / 1234(99.8 \%)$ & $2 / 1234(0.16 \%)$ & Deleción $2 \mathrm{pb}$ \\
IV & 3 & 1252 & $1232 / 1234(99.8 \%)$ & $2 / 1234(0.16 \%)$ & Inserción $2 \mathrm{pb}$ \\
VI & 12 & 993 & $1232 / 1234(80.3 \%)$ & $2 / 1234(0.16 \%)$ & Deleción $240 \mathrm{pb}$ \\
\hline
\end{tabular}


KX943034. A. m. scutellata x A.m. capensis (Sur de África)

A. m. scutellata $\times$ A. m. capensis C51

A. m. scutellata $\times$ A. m. capensis C51

A. m. scutellata $\times$ A. m. capensis $C 65$

A. m. scutellata $\times$ A. m. capensis $\mathrm{C} 23$

A. m. scutellata $\times$ A. m. capensis $\mathrm{C} 45$

A. m. scutellata $\times$ A. m. capensis $\mathrm{C} 45$

A. m. scutellata $\times$ A. m. capensis C36

A. m. scutellata $\times$ A. m. capensis C36

KX870183. A. m. capensis(Sur de África)

KJ601784. A. m. scutellata (Querétaro, Méx.)

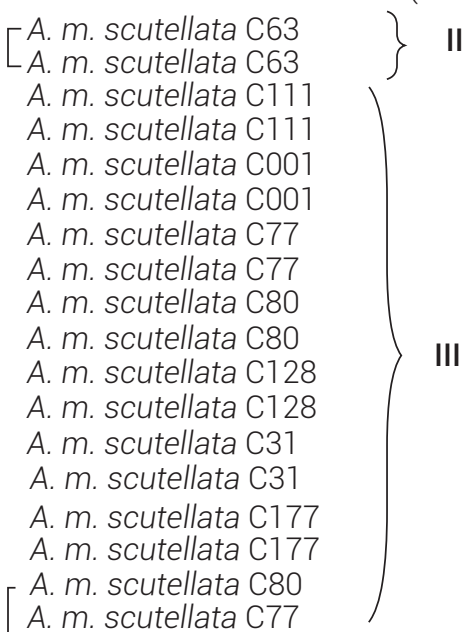

$\left.\begin{array}{l}\text { A. m. scutellata C77 } \\ \text { KT279826. A. m. scutellata (California, EUA) }\end{array}\right\}$ IV

A. m. scutellata $\mathrm{C} 60$

A. m. scutellata C60

A. m. scutellata $\mathrm{C001}$

A. m. scutellata C001

A. m. scutellata C60

A. m. scutellata C60

A. m. scutellata C80

A. m. scutellata C128

A. m. scutellata C128

A. m. scutellata C31

A. m. scutellata C60

A. m. scutellata C80

KX463841. A. m. iberiensis (Portugal)

KY926883. A. m. intemisa (Marruecos, África) $\}$ v

KY926884. A. m. mellifera (Noruega)
A. m. scutellata C63
A. m. scutellata C63
A. m. scutellata $\mathrm{C} 187$
A. m. scutellata C57
A. m. scutellata C57
A. m. scutellata C74
A. m. scutellata C74
A. m. scutellata C187
A. m. scutellata C56
A. m. scutellata C57
A. m. scutellata C089
A. m. scutellata C089

III Bombus terrestris 
El haber encontrado estos dos tipos de germoplama, de A. m. scutellata y del híbrido A. m. scutellata $\times$ A. $m$. capensis pudiera explicarse por el hecho de que las seis reinas africanizadas con las que se formó la población euroafricana fue el resultado de la captura de 177 enjambres africanizados (Pérez-Sato y Cervantes-Santana, 2001) de lo que venía migrando desde su escape de Sao Paulo, Brasil en 1957 (Kerr, 1967; Padilla et al., 1992). Según lo descrito, el germoplasma africanizado fue colectado de enjambres en Tabasco, parte de Campeche y Veracruz entre 1987 y 1989, justo después de la entrada de la abeja A. m. scutellata a México (Moffett et al., 1987). El origen geográfico de las abejas que llegaron a Brasil fue Tabora, Tanzania, y Pretoria y Johannesburgo, Sudáfrica (Nogueira-Neto, 1964). A. m. scutellata tiene una distribución geográfica en el centro y el oeste de África, mientras que A. m. capensis se localiza al sur de Sudáfrica (Beekman et al. 2008), por lo que es probable que al menos alguna de las reinas introducidas a Brasil haya sido variante híbrida de A. m. scutellata × A. m. capensis, de Sudáfrica.

La población de estudio se formó con reinas vírgenes de origen africanizado, seleccionadas por mansedumbre y criterios apícolas, que se cruzaron con zánganos de origen europeo también seleccionados (Pérez-Sato y Cervantes-Santana, 2001). El propósito de realizar la recombinación genética de esa manera fue el aprovechamiento de atributos apícolas deseables de ambos tipos de abejas (Guzmán-Novoa et al., 2011); de las africanizadas, se buscaron atributos tales como alta postura de reinas, tolerancia a enfermedades (entre ellas varroasis), alta producción de polen y propóleo; así mismo, con la selección hacia mansedumbre, sedentarismo y otros criterios de importancia apícola, antes de la recombinación genética, se habían contrarrestado efectos negativos como alta tendencia a enjambrar, pillaje y alto comportamiento defensivo que implicaba alto costo de manejo de colmenas (Guzmán-Novoa et al., 2011); con la recombinación con zánganos europeos se trató de aprovechar la capacidad productiva de miel, mansedumbre y uniformidad de postura. El resultado ha sido favorable debido a que el comportamiento defensivo es altamente heredable (Guzmán-Novoa y Page, 1992; 1993; 1999; Stort 1975) y puede ser determinado por efectos de dominancia genética (Degrandi-Hoffman et al., 1998; Guzmán-Novoa y Page, 1994b; Guzmán-Novoa et al., 2002; Stort, 1975) y los efectos paternos hacia mansedumbre son más relevantes que los maternos (Degrandi-Hoffman et al., 1998; GuzmánNovoa et al., 2002).

Es conocido que las abejas africanizadas (A. $m$. scutellata) son más resistentes a varroa (De Jong, 1997; Guzmán-Novoa et al., 1996; Mondragón et al., 2006) por su conducta higiénica y de acicalamiento, además de la baja fertilidad del ácaro en las celdas de cría de obreras (Arechavaleta-Velasco y Guzmán-Novoa, 2001; Calderón et al., 2010; Guzmán-Novoa et al., 1999; Vandame et al., 2002).

Es conveniente resaltar que, desde su establecimiento como población recombinante de abejas africanizadas con europeas y hasta la fecha, el apiario experimental del Colegio de Postgraduados es una de las pocas poblaciones experimentales que muestran tolerancia natural a varroa y se ha mantenido sin aplicar acaricidas, lo cual puede ser atribuible a la contribución del germoplasma africanizado

Cuadro 2. Diferencia en polimorfismo en secuencias concatenadas de los espacios intergénico COI-COIl y ND5 de testigos incluidos en el análisis filogenético.

\begin{tabular}{|c|c|c|c|c|}
\hline Testigos & Longitud (pb) & $\begin{array}{c}\text { Proporción de sitios } \\
\text { conservados }\end{array}$ & $\begin{array}{l}\text { Cantidad de sitios } \\
\text { variables(\%) }\end{array}$ & $\begin{array}{c}\text { Inserciones/deleciones con } \\
\text { respecto a A. m. scutellata } \\
\text { KJ601784 }\end{array}$ \\
\hline $\begin{array}{l}\text { A. m. capensis } \\
\text { KX870183 }\end{array}$ & 1042 & 1038/1233 (84.18 \%) & 2/1233 (0.16 \%) & Deleción 191 pb \\
\hline $\begin{array}{l}\text { A. m. mellifera } \\
\text { KY926884 }\end{array}$ & 1069 & 1035/1232 (84 \%) & 33/1232 (2.6 \%) & Deleción 163 pb \\
\hline $\begin{array}{l}\text { A. m. iberiensis } \\
\text { KX463941 }\end{array}$ & 1261 & $1211 / 1277$ (94.83 \%) & 4/1277 (0.32 \%) & Inserción 44 pb \\
\hline $\begin{array}{l}\text { A. m. intermisa } \\
\text { KY926883 }\end{array}$ & 1072 & 1046/1231 (84.97 \%) & 26/1231 (2.11\%) & Deleción 159 pb \\
\hline $\begin{array}{l}\text { A. m. scutellata } \\
\text { KT279826 }\end{array}$ & 1034 & 1229/1234 (99.59 \%) & 2/1234 (0.16\%) & Inserción 2 pb \\
\hline $\begin{array}{l}\text { A. m. scutellata X A. m. } \\
\text { capensis KX943034 }\end{array}$ & 1041 & 1040/1232 (84.4 \%) & 0 & Deleción 191 pb \\
\hline
\end{tabular}


y con capacidad productiva de miel sobresaliente como contribución del germoplasma europeo.

El hecho de haber encontrado solamente $\operatorname{ADN}_{\mathrm{mt}}$ de origen africano en el estudio parece indicar que las abejas africanizadas, en su dispersión desde el escape en Brasil en 1957 y hasta su establecimiento en México, han conservado el material genético mitocondrial de origen africano.

\section{CONCLUSIONES}

El análisis de $\mathrm{ADN}_{\mathrm{mt}}$ confirma que el linaje materno del apiario del Colegio de Postgraduados, Montecillo, Estado de México corresponde al africano (A), como se infería de antecedentes del establecimiento de dicha población. Los resultados indican que $74 \%$ de las colmenas analizadas corresponden a la subespecie A. m. scutellata y $26 \%$ al híbrido A. m. scutellata $\times$ A. m. capensis.

\section{BIBLIOGRAFÍA}

Arechavaleta-Velasco M. E. and E. Guzmán-Novoa (2001) Relative effect of four characteristics that restrain the population growth of the mite Varroa destructor in honey bee (Apis mellifera) colonies. Apidologie 32:157-174, https://doi.org/10.1051/apido:2001121

Beekman M., M. H. Allsopp, T. C. Wossler and B. P. Oldroyd (2008) Factors affecting the dynamics of the honeybee (Apis mellifera) hybrid zone of South Africa. Heredity 100:13-18, https://doi. org/10.1038/sj.hdy.6801058

Bouga M., P. C. Harizanis, G. Kilias and S. Alahiotis (2005) Genetic divergence and phylogenetic relationships of honey bee Apis mellifera (Hymenoptera: Apidae) populations from Greece and Cyprus using PCR-RFLP analysis of three mtDNA segments Apidologie 36:335-344, https://doi.org/10.1051/apido:2005021

Calderón R. A., J. W. van Veen, M. J. Sommeijer and L. A. Sánchez (2010) Reproductive biology of Varroa destructor in Africanized honey bees (Apis mellifera). Experimental and Applied Acarology 50:281-297, https://doi.org/10.1007/s10493-009-9325-4

Clarke K. E., T. E. Rinderer, P. Franck, J. G. Quezada-Euán and B. P. Oldroyd (2002) The africanization of honeybees (Apis mellifera L.) of the Yucatan: a study of a massive hybridization event across time. Evolution 56:1462-1474, https://doi. org/10.1111/j.0014-3820.2002.tb01458.x

De Jong D. (1997) Mites: varroa and other parasites of brood. In: Honey Bee Pests, Predators, and Diseases. R. A. Morse and K. Flottum (eds.). 3rd edition. A. I. Root Publishing. Medina, Ohio, USA pp:279-327.

Degrandi-Hoffman G., A. Collins, J. H. Martin, J. O. Schmidt, and H. G. Spangler (1998) Nest defense behavior in colonies from crosses between Africanized and European honey bees (Apis mellifera L.) (Hymenoptera: Apidae). Journal of Insect Behavior 11:37-45, https://doi.org/10.1023/A:1020862432087

Doyle J. J. and J. L. Doyle (1987) A rapid DNA isolation procedure from small quantities of fresh leaf tissue. Phytochemical Bulletin 19:11-15

Garnery L., J. M. Cornuet and M. Solignac (1992) Evolutionary history of the honey bee Apis mellifera inferred from mitochondrial DNA analysis. Molecular Ecology 1:145-154, https://doi. org/10.1111/j.1365-294X.1992.tb00170.x

Gary N. E., H. V. Daly, S. Locke and M. Race (1985) The Africanized honeybee: ahead of schedule. California Agriculture 39:4-7.

Guzmán-Novoa E., A. Correa B., L. G. Espinosa M. and G. Guzmán N. (2011) Colonization, impact and control of Africanized honey bees in Mexico. Veterinaria México 42:149-178.
Guzmán-Novoa E., G. J. Hunt, J. L. Uribe, C. Smith and M. E. ArechavaletaVelasco (2002) Confirmation of QTL effects and evidence of genetic dominance of honeybee defensive behavior: results of colony and individual behavioral assays. Behavior Genetics 32:95-102, https://doi.org/10.1023/A:1015245605670

Guzmán-Novoa E. and R. E. Page (1992) Breeding Honey Bees in Africanized Areas. In: Apiculture for the 21st Century. R Hoopingarner and L. J. Connor (eds.). Wicwas Press. Cheshire, Connecticut, USA pp:12-14

Guzmán-Novoa E. and R. E. Page (1993) Backcrossing Africanized honey bee queens to European drones reduces colony defensive behavior. Annals of the Entomological Society of America 86:352-355, https://doi.org/10.1093/aesa/86.3.352

Guzmán-Novoa E. and R. E. Page (1994a) The impact of Africanized bees on Mexican beekeeping. American Bee Journal 134:101-106.

Guzmán-Novoa E. and R. E. Page (1994b) Genetic dominance and worker interactions affect honeybee colony defense. Behavioral Ecology 5:91-97, https://doi.org/10.1093/beheco/5.1.91

Guzmán-Novoa E., A. Sánchez, R. E. Page and T. García (1996) Susceptibility of European and Africanized honeybees (Apis mellifera L.) and their hybrids to Varroa jacobsoni Oud. Apidologie 27:93-103.

Guzmán-Novoa E. and R. E. Page (1999) Selective breeding of honey bees (Hymenoptera: Apidae) in Africanized areas. Journal of Economic Entomology 92:521-525, https://doi.org/10.1093/ jee/92.3.521

Guzmán-Novoa E., R. Vandame and M. E. Arechavaleta (1999) Susceptibility of European and Africanized honey bees (Apis mellifera L.) to Varroa jacobsoni Oud. in Mexico. Apidologie 30:173-182 https://doi.org/10.1051/apido:19990207

Hall T. A. (1999) BioEdit: a user-friendly biological sequence alignment editor and analysis program for Windows 95/98/NT. Nucleic Acids Symposium Series 41:95-98.

Hall H. G. and K. Muralidharan (1989) Evidence from mitochondrial DNA that African honey bees spread as continuous maternal lineages. Nature 339:211-213, https://doi.org/10.1038/339211a0

Kerr W. E. (1967) The history of the introduction of African bees to Brazil South African Bee Journal 39:3-5.

Magnus R. M., A. D. Tripodi and A. L. Szalanski (2014) Mitochondrial DNA diversity of honey bees (Apis mellifera) from unmanaged colonies and swarms in the United States. Biochemical Genetics 52:245-257, https://doi.org/10.1007/s10528-0149644-y

Moffett J. O., D. L. Maki, T. Andre and M. M. Fierro (1987) The Africanized bee in Chiapas, Mexico. American Bee Journal 127:517-520.

Mondragón L., S. Martin and R. Vandame (2006) Mortality of mite offspring: a major component of Varroa destructor resistance in a population of Africanized bees. Apidologie 37:67-74, https:// doi.org/10.1051/apido:2005053

Nogueira-Neto P. (1964) The spread of a fierce African bee in Brazil. Bee World 45:119-121, https://doi.org/10.1080/000577 2X.1964.11097060

Özdil F. and F. İlhan (2012) Genetic divergence of Turkish Apis mellifera subspecies based on sequencing of ND5 mitochondrial segment. Sociobiology 59:225-234.

Padilla F., F. Puerta, J. M. Flores and M. Bustos (1992) Bees, apiculture and the new world. Archivos de Zootecnia 41:563-567.

Pérez-Sato J. A. y T. Cervantes-Santana (2001) Selección para tolerancia a Varroajacobsoni Oud. en una población de abejas euroafricanas. Agrociencia 35:413-421.

Rahimi A. (2015) Study of the genetic diversity of Iranian honey bee (Apis mellifera meda Skorikow, 1829) populations using the mtDNA COI-COII intergenic region. Biologija 61:54-59, https:// doi.org/10.6001/biologija.v61i2.3139

Ronquist F. and J. P. Huelsenbeck (2003) MrBayes 3: Bayesian phylogenetic inference under mixed models. Bioinformatics 19:1572-1574, https://doi.org/10.1093/bioinformatics/btg180

Ruttner F. (1988) Biogeography and Taxonomy of Honeybees. SpringerVerlag. Heidelberg, Berlin. 284 p, https://doi.org/10.1007/9783-642-72649-7

Schneider S. S., G. DeGrandi-Hoffman and D. R. Smith (2004) The African honey bee: factors contributing to a successful biological invasion. Annual Review of Entomology 49:351-376, https:// doi.org/10.1146/annurev.ento.49.061802.123359 
Seeley T. D. (2016) Honeybee Ecology: A Study of Adaptation in Social Life. Princeton University Press. Princeton, New Jersey, USA. $214 \mathrm{p}$.

Stort A. C. (1974) Genetic study of aggressiveness of two subespecies of Apis mellifera in Brazil 1. Some tests to measure aggressiveness. Journal of Apicultural Research 13:33-38, https://doi.org/10.1080/00218839.1974.11099756

Stort A. C. (1975) Genetical study of the aggressiveness of two subspecies of Apis mellifera in Brazil. V. Number of stings in the leather ball. Journal of the Kansas Entomological Society 48:381-387.

Sugden D. A. and K. R. Williams (1991) October 15: the day the bee arrived. Gleanings in Bee Culture 119:18-21.

Tamura K., G. Stecher, D. Peterson, A. Filipski and S. Kumar (2013) MEGA6: molecular evolutionary genetics analysis version 6.0. Molecular Biology and Evolution 30:2725-2729, https://doi.org/10.1093/ molbev/mst197

Thompson J. D., D. G. Higgins and T. J. Gibson (1994) CLUSTAL W: improving the sensitivity of progressive multiple sequence alignment through sequence weighting, positions-specific gap penalties and weight matrix choice. Nucleic Acids Research 22:4673-4680.

Valido A., M. C. Rodríguez-Rodríguez y P. Jordano (2014) Impacto de la introducción de la abeja doméstica (Apis mellifera, Apidae) en el Parque Nacional del Teide (Tenerife, Islas Canarias) Ecosistemas 23:58-66, https://doi.org/10.7818/ECOS.2014.233.08

Vandame R., S. Morand, M. E. Colin and L. P. Belzunces (2002) Parasitism in the social bee Apis mellifera: quantifying costs and benefits of behavioral resistance to Varroa destructor mites. Apidologie 33:433-445, https://doi.org/10.1051/apido:2002025

Winston M. L. (1992) Killer Bees: The Africanized Honey Bee in the Americas. Harvard University Press. Cambridge, Massachusetts, USA. 162 p.

Zhang Z., S. Schwartz, L. Wagner and W. Miller (2000) A greedy algorithm for aligning DNA sequences. Journal of Computational Biology 7:203-214, https://doi.org/10.1089/10665270050081478 